\title{
Obesity Management In Primary Care: Assessment of Readiness to Change Among 284 Family Practice Patients
}

\author{
Everett Logue, PbD, Karen Sutton, $\mathrm{PbD}$, David Jarjoura, $\mathrm{PbD}$, and \\ William Smucker, MD
}

Background: Most adults in primary care are overweight or obese; two thirds of patients with weight problems have other obesity-related conditions. The study objective was to explore the feasibility of a primary care obesity intervention based on the transtheoretical model (TM) of behavior change and principles of chronic disease (CD) care.

Methods: A prospective study of the initial version of the TM-CD intervention with obese family practice patients $(n=284)$ yielded cross-sectional data on baseline stage of change for six target behaviors: dietary fat, portion control, vegetable intake, fruit intake, usual physical activity, and planned exercise. The sample consisted of obese patients scheduled for an office visit during times when recruitment and informed consent did not conflict with acute care.

Results: Obese patients volunteering for a TM-CD program are in different stages of change for six target behaviors. Preparation was the most frequently reported stage for increased exercise (49\%) or activity (34\%), decreased dietary fat consumption (44\%), and increased portion control (51\%). Patients in a particular stage for one behavior were distributed across all five stages for another behavior. Stage of change for five target behaviors was associated with body mass index or waist girth $(P<.05)$ in a manner consistent with stage-of-change theory.

Conclusions: Using the transtheoretical model of behavior change will allow physicians to recognize when obese patients are receptive to specific behavioral interventions. (J Am Board Fam Pract 2000;13: 164-71.)

The National Institutes of Health (NIH) recently announced what many primary care physicians have known for years-that most adults in this country $(55 \%)$ are overweight (body mass index between 25.0 and 29.9) or obese (body mass index of 30.0 or more). 'Two thirds of patients with weight problems are also likely to have hypertension, hyperlipidemia, diabetes, coronary artery disease, or another obesity-related comorbid condition. ${ }^{1}$ Because most obese patients are initially seen in primary care, obese

Submitted, revised 25 October 1999.

From the Department of Family Practice (EL, KS, WS), Summa Health System, Akron; the Department of Psychology (KS), Oberlin College, Oberlin; and the Division of Community Health Sciences (DJ), Northeastern Ohio Universities College of Medicine, Rootstown, Ohio. Address reprint requests to Everett Logue, $\mathrm{PhD}$, Department of Family Practice, Summa Health System, 525 East Market Street, Suite 290, Akron, OH 44309-2090.

This research was supported by grants from the Summa Health System Foundation and the Agency for Health Care Research and Quality (R01-HS 08803-02). patients are encountered during a substantial percentage of office visits. ${ }^{2}$ Thus, obesity and its sequelae are serious challenges to primary care and have implications for both chronic disease and practice management. ${ }^{1-3}$

Although obesity poses a serious health threat, and obesity-related illnesses strain health care resources, few obese primary care patients receive effective treatment. ${ }^{4-7}$ Primary care physicians might offer a brief educational encounter consisting of a lecture on the risks of obesity and might advise obese patients to eat less and exercise more. Time constraints of primary care, however, often prevent discussion of how to implement the eatless, exercise-more prescription and can limit the physician's opportunity to consider the patient's emotional status or to apply principles of behavior change to patients' eating and exercise habits. ${ }^{8}$ Additional obstacles include the reluctance of insurance companies to reimburse physicians for obesity 
counseling and management, and the cost and time required to provide adequate follow-up care for obese patients. In light of the realities of primary care, it is understandable that physicians undertreat obesity. ${ }^{1,4,8}$

Some primary care physicians refer obese patients to specialized obesity treatment programs., 90 These programs, which usually include short-term or fixed-duration therapy groups at a clinical center, teach a variety of dieting and lifestyle skills (physical activity, stress management) under the direction of weight-loss counselors. Although several of these specialized interventions have been shown to be effective, particularly those involving cognitive-behavioral counseling and physical activity, they also have serious limitations. Because these programs are usually offered at a centralized location and require the patient to travel to the weight-loss center, many obese patients never find their way to a treatment group. For those who do, the autonomy of the treatment program discourages communication between weight-loss counselors and primary care physicians. ${ }^{1}$ Furthermore, even the most effective fixedlength obesity treatment program fails to make an impact on the long-term health of $75 \%$ of patients, because the initial weight loss is followed by relapse and weight regain. ${ }^{11,12}$

The NIH guideline recommends treatment for obesity. The guideline, however, does not describe how treatment can be feasibly implemented in primary care practice. ${ }^{1}$ In light of the problems described above, we reasoned that for an obesity treatment to be truly effective, it would have to be readily available to all patients, integrated into the patient's other medical treatment, cost-effective, and efficacious in the long-term.

One strategy for managing obesity in primary care that meets these criteria involves viewing obesity as a chronic disease. ${ }^{1}$ Because the origin and course of obesity are similar to a variety of chronic conditions, it seems more reasonable to try to prevent or manage obesity rather than to cure it with a single session of advice or a short-term program. ${ }^{13}$ Managing obesity as a chronic condition would involve the same components of primary care used successfully with other chronic conditions: periodic reassessments, life-span access to counseling and other treatments, and continuity of care by health care personnel who are aware of the patient's biopsychosocial history.
Obese patients differ in their commitment to weight loss as well as their ability to make the complex behavioral and cognitive changes required for life-long weight control. ${ }^{12-14}$ Nonetheless, many formal obesity treatments rely on uniform dietary, exercise, or cognitive-behavioral protocols. We hypothesized that a treatment would be more effective if it could be tailored to the patient's readiness and ability to change target behaviors. This hypothesis is based on the transtheoretical model of behavior change. ${ }^{14}$ The transtheoretical model assumes that patient readiness to make changes on relevant behaviors can be assessed or assigned to a stage, and that treatment efficacy can be enhanced by applying the cognitive and behavioral change processes that are best suited to the patient's stage. ${ }^{14}$

After recognizing that obesity is a complex, chronic condition and that obese patients are highly diverse, we hypothesized that obese patients would benefit most from treatment that combines the principles of chronic disease management in primary care with the transtheoretical model of behavior change. ${ }^{14-16}$ The general purpose of the study summarized in this report was to investigate the feasibility of a primary care intervention for obesity based on a combined transtheoretical modelchronic disease (TM-CD) paradigm. More specifically, we attempted to measure stage of change for six target behaviors in a sample of obese primary care patients, because the model dictated that this step is first in tailoring treatments. One objective of the study was to explore the heterogeneity of stages for the six target behaviors. A second objective was to examine the relations between anthropometric indices and the stages of change.

\section{Methods}

The study enrolled 284 obese patients, aged 30 to 69 years. Study patients were recruited from a community-hospital-affiliated family practice center (FPC) by nursing staff and physicians. The distribution of patients, problems, and diagnoses at this FPC reflects those seen in samples from community practices across the country. ${ }^{17}$

\section{Sample Selection}

Female patients with a body mass index (BMI) greater than $27.3 \mathrm{~kg} / \mathrm{m}^{2}$ or a waist-hip ratio greater than 0.800 and male patients with a BMI greater 
than $27.8 \mathrm{~kg} / \mathrm{m}^{2}$ or a waist-hip ratio greater than 0.950 were eligible for the program. ${ }^{18,19}$ Nurses approached eligible patients for consent after they were weighed at their scheduled acute or follow-up office visits. Physicians referred eligible patients after they were seen in the examination rooms. Patients were not approached or recruited when they were acutely ill, when the staff were busy, or when we had exceeded our monthly quota of 200 participants. Less than one third of the age- and BMI-eligible FPC patients seen between January 1996 and April 1997 were enrolled in the feasibility study.

\section{Data Collection}

Once recruitment and informed consent were completed, nurses took girth measurements and administered a staging questionnaire. Using the assessment strategy developed by Prochaska and colleagues, ${ }^{20}$ patients chose one of five statements that best described their readiness to change each of six weight-related target behaviors. The five statements ranged from "not thinking about changing" to "actively making the change for more than six months." The six target behaviors included increased planned exercise, increased daily activity, increased fruit consumption, increased vegetable consumption, decreased dietary fat, and decreased portion sizes. Thus, a staging profile consisted of a single response for each of the six behaviors.

\section{Statistical Analysts}

Study data were entered, verified, and edited with Epi Info ${ }^{21}$ software or scanned, verified, and edited with Teleform software ${ }^{22}$ before they were exported to SAS, Cary, NC, for analysis. Proportions were compared with chi-square tests. Overlap of staging (precontemplation, contemplation, preparation, action, and maintenance) for the six target behaviors was examined with bivariate tables and then summarized with rank correlations. The relations between anthropometric (BMI, waist girth, or waist-hip ratio) or biologic risk factors (age, systolic blood pressure, diastolic blood pressure) and stage of change for the six target behaviors were assessed by tests for linear trends in means. $P<.05$ was considered significant.

\section{Results}

The mean age of participants was 46.5 years $(\mathrm{SD}=$ 10.3 years), $81 \%$ were female, and the mean BMI was $35.9 \mathrm{~kg} / \mathrm{m}^{2}\left(\mathrm{SD}=7.3 \mathrm{~kg} / \mathrm{m}^{2}\right)$. Figure 1 displays the distribution of BMIs for participants at enrollment. Twenty percent of the patients were overweight $\left(25\right.$ to $\left.29.9 \mathrm{~kg} / \mathrm{m}^{2}\right)$ and $79 \%$ were obese $\left(30.0+\mathrm{kg} / \mathrm{m}^{2}\right)$ based on recent NIH criteria. ${ }^{1}$ Five patients $(1.8 \%$ ) had borderline BMIs (23.0 to 24.9 $\mathrm{kg} / \mathrm{m}^{2}$ ) but elevated waist-hip ratios. The average (median) waist-hip ratio for women was 0.838 (range $=0.684$ to 1.198 ), whereas the average waist-hip ratio for men was 0.955 (range $=0.856$ to 1.177). The average waist girth for women was $102.5 \mathrm{~cm}$ (40.4 in), whereas the average waist girth for men was $109.0 \mathrm{~cm}$ (42.9 in).

Figure 2 displays the distribution of stage of change for the six target behaviors at baseline. For decreased dietary fat, increased portion control, and increased exercise, patients in the contemplation and preparation stages constituted more than $50 \%$ of the sample. This documents the potential for behavior change in these areas, because these obese patients are not yet in action for these key behaviors.

Cross-tabulations of stage of change for paired behaviors (activity and exercise, fat and portion control, fruits and vegetables) revealed heterogeneity in patients' profiles. For example, patients in the preparation stage for increased usual activity $(n=$ 96) were distributed across all five stages of change for increased planned exercise (Table 1). Similarly, patients in the preparation stage for planned exercise $(n=135)$ could be in maintenance (36), action (22), or preparation (74) for increased usual activity. Looking at Table 1 in another way, 57\% (158 of 278) of patients were in the same stage of change for these paired behaviors (were concordant), leaving $43 \%$ who were discordant. This variation shows how obese patients differ with respect to which weight-loss behaviors they are most prepared to work on at any given clinical encounter.

Table 2 shows the rank correlations among stages for the six target behaviors. The largest correlation (0.49) is between the stages for increased activity versus increased exercise (Table 1). All the other rank correlations in Table 2 are smaller than 0.49 , which suggests that less than $25 \%$ of the variation in ranks for one behavior is explained by variation in ranks for another. The relatively small size of the correlations in Table 2 documents the heterogeneity of stages for the six target behaviors.

Table 3 displays the relations between indices of obesity and stage of change for the six target be- 


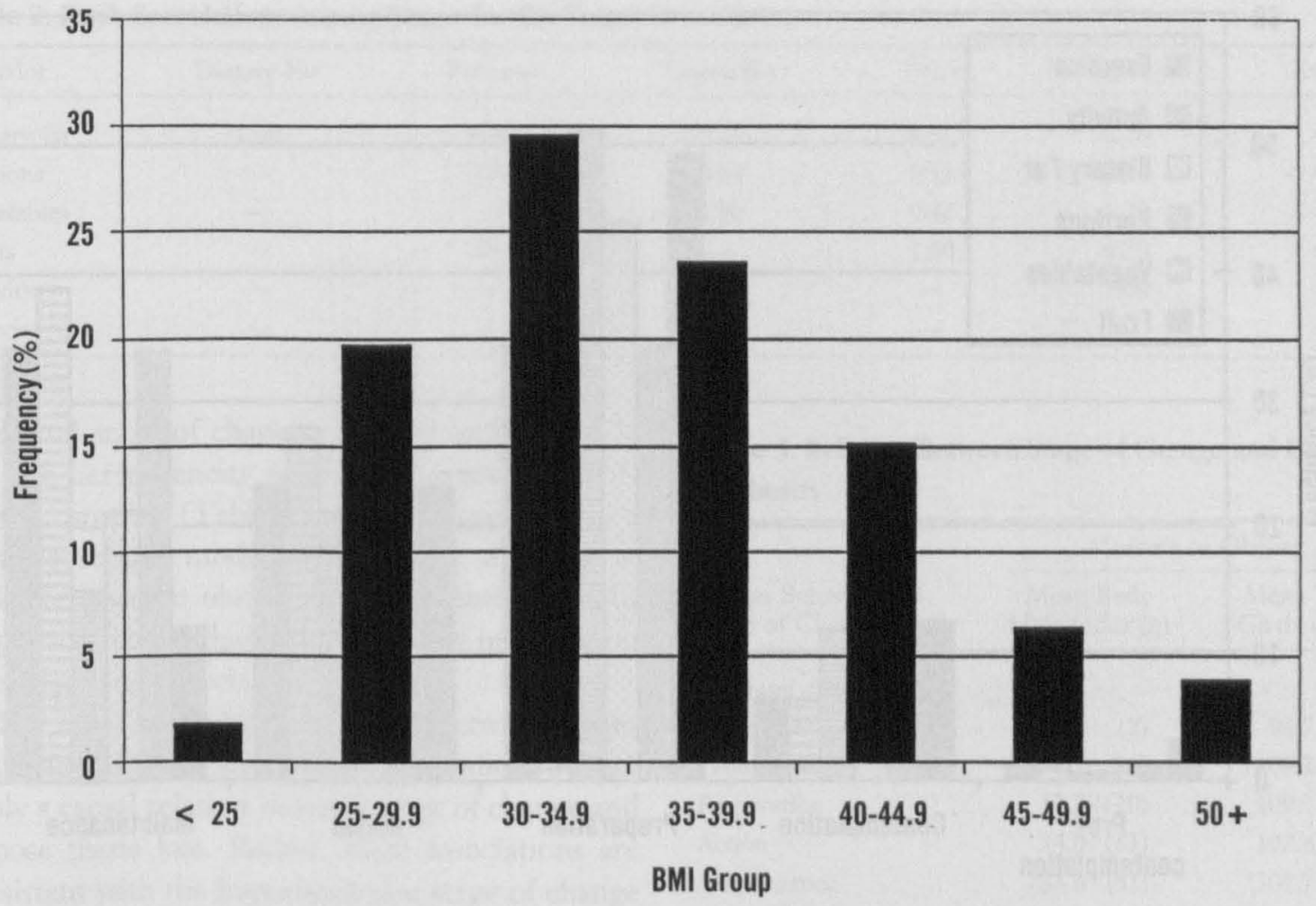

Figure 1. Body mass index (BMI) distribution among study patients. Persons with a BMI of $25.0-29.9 \mathrm{~kg} / \mathrm{m}^{2}$ are generally considered overweight. ${ }^{1}$ Persons with a BMI of $30.0 \mathrm{~kg} / \mathrm{m}^{2}$ or more are generally considered obese.

haviors. Trend tests indicated that both mean BMI and mean waist girth are negatively related to stage of change for five of the six target behaviors: decreased dietary fat $(P=.0003$ and $P=.002)$, increased portion control $(P=.01$ and $P=.002)$, increased activity ( $P=.0001$ for both), increased exercise $(P=.0002$ and $P=.0001)$, and increased fruit intake $(P=.01$ and $P=.02)$. Stage of change for increased vegetable consumption was not significantly associated with either anthropometric index. Put another way, the data indicate that patients in the action or maintenance stages for increased exercise and each of the other four significant behaviors have lower BMIs or smaller waist girths than patients in the contemplation or preparation stages for these behaviors.

Similar trend tests were performed on mean patient age by stage of change. These analyses revealed that older patients were more likely to be in the action or maintenance stages for decreased dietary fat $(P=.005)$, and increased vegetable $(P=$ $.001)$ or fruit intake $(P=.001)$, and less likely to be in the action or maintenance stages for increased exercise $(P=.04)$. There was no relation between stage of change and mean waist-hip ratios or blood pressures.

The patient's sex was not a good indicator of a patient's stage of change. The (univariate) stage distributions were identical for women and men with the exception of increased fruit consumption, for which a larger proportion of women ( $26 \%$ vs $13 \%)$ reported being in action $\left(\chi^{2}[\mathrm{df}=3]=8.4\right.$; $P=.04)$.

Figure 3 illustrates the relation between mean $\mathrm{BMI}$ and activity stage of change reported in Table 3. The stepwise relations between BMI or waist girth and the other target behaviors follow a similar pattern.

\section{Discussion}

According to the transtheoretical model, patients in the action and maintenance stages should be engaging in target behaviors that will reduce excess adipose tissue for the longer term. Patients in preparation are almost ready to change their behavior, while patients in the earliest stages are not ready. Information about patients' stage-of-change profiles could be used by family physicians and other 


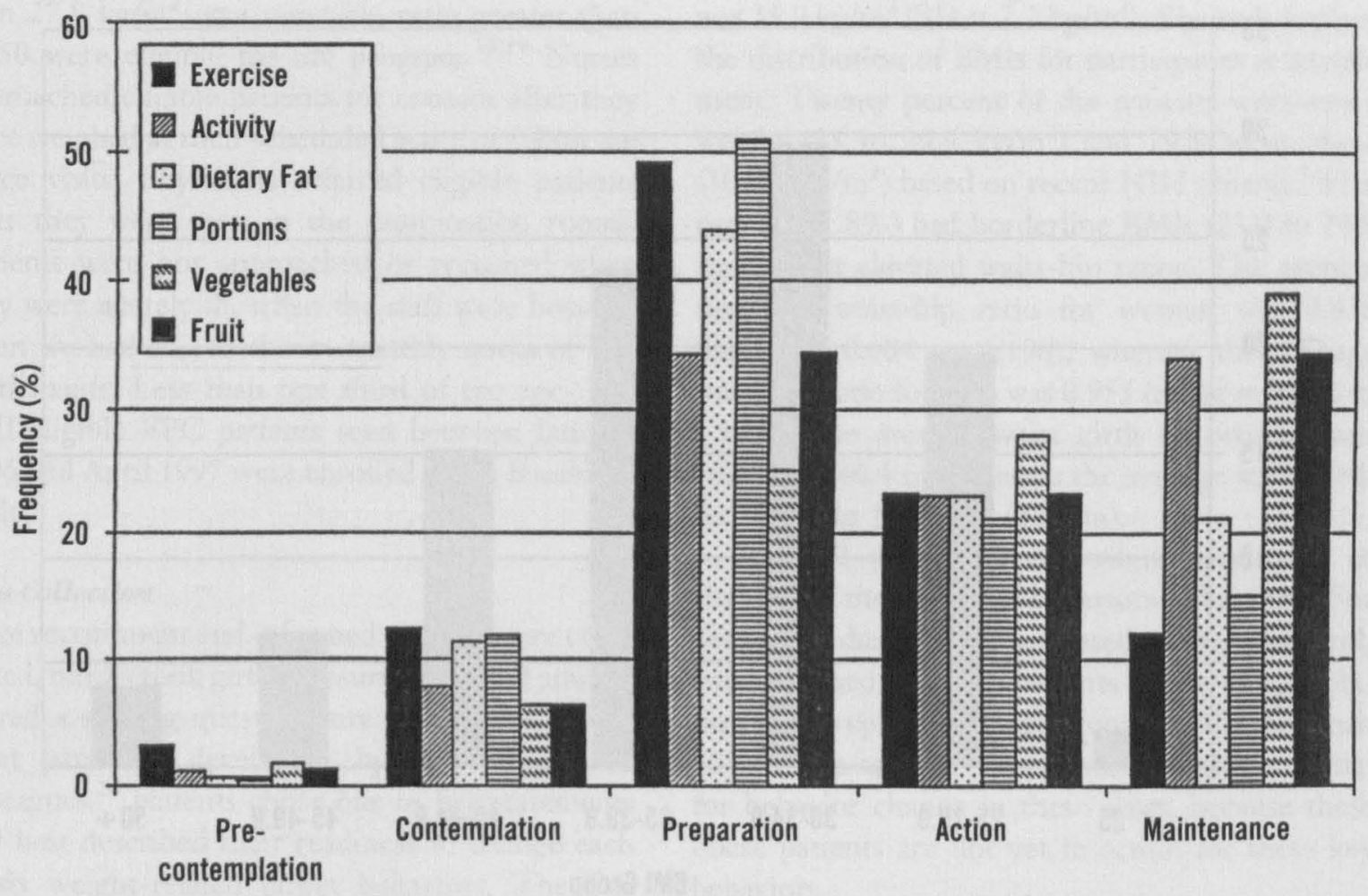

Stage of Change

Figure 2. Baseline stage of change distribution among 284 family practice patients.

clinicians to get the appropriate cognitive-behavioral advice to responsive obese patients at the right time. ${ }^{14,20}$ Thus, the cycle of weight loss and regain might be broken, and patients might avoid losing the same 20 pounds again.

We found that patients in the preparation stage for one target behavior were often not in the preparation stage for other target behaviors. Specifically, rank correlations of stages for one behavior compared with another were generally low. This heterogeneity of stage-of-change profiles is important because it describes subsets of obese patients who should be more or less responsive to different types of cognitive-behavior interventions. ${ }^{14}$ Moreover, this heterogeneity is consistent with clinical experience indicating that obese patients differ in their readiness to change key dietary and exerciserelated behaviors.

We also found that patients in the more advanced stages of change for activity or exercise, fruit intake, dietary fat, and portion control have smaller BMIs or waist girths, whereas patients in the earlier stages have larger BMIs or waist girths. The stepwise relations between anthropometric in-

Table 1. Relation Between Stage of Change for Increased Usual Activity and Increased Planned Exercise Among Study Patients $(\mathrm{n}=\mathbf{2 7 8})$.

\begin{tabular}{|c|c|c|c|c|c|c|}
\hline \multirow{2}{*}{$\begin{array}{l}\text { State of Change for } \\
\text { Increased Usual Activity }\end{array}$} & \multicolumn{5}{|c|}{ Stage of Change for Increased Planned Exercise } & \multirow[b]{2}{*}{ Total } \\
\hline & Precontemplation & Contemplation & Preparation & Action & Maintenance & \\
\hline Precontemplation & 1 & 0 & 2 & 0 & 0 & 3 \\
\hline Contemplation & 2 & 16 & 1 & 2 & 0 & 21 \\
\hline Preparation & 2 & 10 & 74 & 8 & 2 & 96 \\
\hline Action & 0 & 5 & 22 & 37 & 1 & 65 \\
\hline Maintenance & 4 & 3 & 36 & 20 & 30 & 93 \\
\hline Total & 9 & 34 & 135 & 67 & 33 & 278 \\
\hline
\end{tabular}


Table 2. Rank Correlations Among Stages for Six Target Behaviors.

\begin{tabular}{lcccccc}
\hline Behavior & Dietary Fat & Portions & Vegetables & Fruit & Activity & Exercise \\
\hline Dietary fat & 1.00 & 0.46 & 0.29 & 0.41 & 0.28 & 0.28 \\
Portions & - & 1.00 & 0.24 & 0.35 & 0.34 & 0.22 \\
Vegetables & - & - & 1.00 & 0.42 & 0.27 & 0.04 \\
Fruits & - & - & - & 1.00 & 0.20 & 0.16 \\
Activity & - & - & - & - & 1.00 & 0.49 \\
Exercise & - & - & - & - & - & 1.00 \\
\hline
\end{tabular}

dices and stage of change (Table 3 and Figure 3) and the heterogeneity of obese patients' stage-ofchange profiles (Tables 1 and 2) suggest that a transtheoretical model intervention directed at multiple, specific obesity-related behaviors might be more effective than the traditional prescription to eat less and exercise more.

The cross-sectional associations between stages of change and the anthropometric indices do not imply a causal relation between stage of change and adipose tissue loss. Rather, these associations are consistent with the hypothesis that stage of change is a surrogate classification of patients' cognitions (intentions) and underlying weight-loss (or weightgain) behaviors (ie, using smaller dinner plates, parking further from destinations, and so on). These underlying behaviors produce the energy surplus or deficit that produces adipose tissue gains or losses. Despite the cross-sectional nature of our data, the stage-of-change algorithm should provide a heuristic for recognizing those subgroups of patients who are more (or less) ready to comply with specific advice.

The patient characteristics and the acute medical care processes that influenced patient enrollment in this study could have distorted the empirical stage-of-change distributions (Figure 2) relative to those obtained from a true random sample of all eligible obese patients. These selection processes, however, should have a small effect on the relation among stages and between stages and mean BMI or waist girth.

Because periodic reassessment is a hallmark of chronic disease care, is it sufficient to measure periodically the patient's weight and stage of change for six target behaviors, or should clinicians also try to estimate calories consumed and burned from patient recalls or questionnaires? ${ }^{23-25}$ One problem with the traditional eat-less, exercise-more prescription is that consuming fewer calories and burning more calories are complex macro-behav-
Table 3. Relation Between Stage of Change and Degree of Obesity

\begin{tabular}{|c|c|c|}
\hline \multirow[b]{2}{*}{$\begin{array}{l}\text { Target Behavior and } \\
\text { Stage of Change }\end{array}$} & \multicolumn{2}{|c|}{ Measure of Obesity } \\
\hline & $\begin{array}{c}\text { Mean Body } \\
\text { Mass Index (n) }\end{array}$ & $\begin{array}{l}\text { Mean Waist } \\
\text { Girth, cm (n) }\end{array}$ \\
\hline \multicolumn{3}{|l|}{ Decreased dietary fat } \\
\hline Precontemplation & $34.5 \quad(2)$ & 94.7 \\
\hline Contemplation & $37.1 \quad(31)$ & $109.7(31)$ \\
\hline Preparation & $37.7(120)$ & $109.1(120)$ \\
\hline Action & $34.0 \quad(63)$ & $102.6(62)$ \\
\hline Maintenance & $\star 33.6 \quad(61)$ & ${ }^{*} 101.7(58)$ \\
\hline \multicolumn{3}{|l|}{ Increased portion control } \\
\hline Precontemplation & $34.0 \quad(2)$ & 112.0 \\
\hline Contemplation & $37.4 \quad(32)$ & $111.4(32)$ \\
\hline Preparation & $36.6(138)$ & $107.0(136)$ \\
\hline Action & $35.0(58)$ & $105.5(57)$ \\
\hline Maintenance & $\star 33.9(43)$ & ${ }^{\star 100.3 \quad(42)}$ \\
\hline \multicolumn{3}{|l|}{ Increased vegetables } \\
\hline Precontemplation & $34.9 \quad(5)$ & $107.0 \quad(5)$ \\
\hline Contemplation & $38.3(17)$ & $109.9(17)$ \\
\hline Preparation & $35.5(70)$ & $104.0(69)$ \\
\hline Action & $36.0(77)$ & $107.8(74)$ \\
\hline Maintenance & $35.6(108)$ & $105.4(108)$ \\
\hline \multicolumn{3}{|l|}{ Increased fruit } \\
\hline Precontemplation & $37.0 \quad(4)$ & $112.3 \quad(4)$ \\
\hline Contemplation & 39.8 (17) & $112.4(17)$ \\
\hline Preparation & $36.4(95)$ & $107.1 \quad(94)$ \\
\hline Action & $35.9(65)$ & $106.0(63)$ \\
\hline Maintenance & *34.6 (96) & ${ }^{\star} 103.5 \quad(95)$ \\
\hline \multicolumn{3}{|l|}{ Increased activity } \\
\hline Precontemplation & $43.6 \quad(3)$ & $119.5 \quad(3)$ \\
\hline Contemplation & $39.2(21)$ & $113.2(21)$ \\
\hline Preparation & $38.1 \quad(95)$ & $110.6(94)$ \\
\hline Action & $35.6(65)$ & $104.6(63)$ \\
\hline Maintenance & *32.8 (93) & ${ }^{*} 100.3(92)$ \\
\hline \multicolumn{3}{|l|}{ Increased exercise } \\
\hline Precontemplation & $35.5 \quad(9)$ & 104.8 \\
\hline Contemplation & 37.9 (34) & $112.7(34)$ \\
\hline Preparation & $37.1(134)$ & $108.1(134)$ \\
\hline Action & $34.3 \quad(67)$ & $102.4(63)$ \\
\hline Maintenance & *31.9 (33) & $98.0 \quad(33)$ \\
\hline
\end{tabular}

${ }^{\star} P<.05$ from a test for trend in means. 


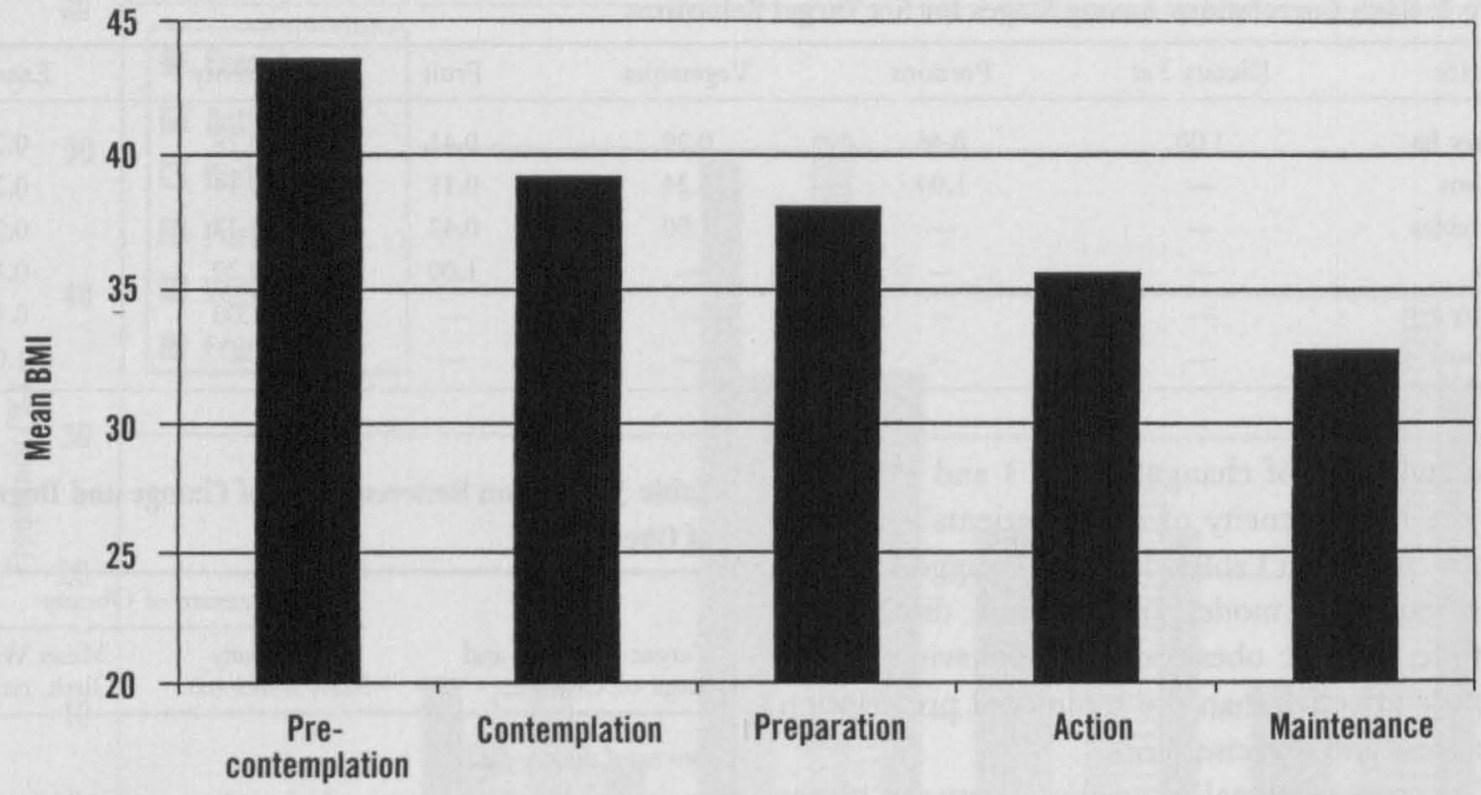

Figure 3. Mean body mass index (BMI) by activity stage of change.

iors. We partitioned consuming fewer calories into four target behaviors (decreased dietary fat, decreased portions, more fruit, more vegetables) and burning more calories into two targets (increased planned exercise, increased usual activity). More research is needed to ascertain the degree of behavioral partitioning that is most consistent with optimal transtheoretical-chronic disease management of obesity.

The heterogeneity of the stage-of-change profiles revealed in our patient sample (Tables 1 and 2) is consistent with other reports of the diversity of obese patients and suggests that treatment individualized to each patient's unique profile might enhance the likelihood of success. ${ }^{16}$ The observation that obese patients in the early stages of change for increased exercise were older is consistent with reports of more sedentary behavior among older individuals. ${ }^{26}$ The observation that obese patients in the late stages of change for decreased dietary fat and increased fruit intake were older is also consistent with the literature. ${ }^{27}$ Because the stage distributions for five of six target behaviors (the exception was fruit consumption) were similar for men and women, it seems reasonable to conclude that obese male primary care patients are as ready as obese female patients when the goal is behavior change to improve health.

Taken as a whole, the data from this feasibility study document the potential of integrating long- term transtheoretical-chronic disease obesity management programs into primary care. More specifically, the data provide preliminary support for the wisdom of tailoring treatments to individual patients' needs. Our experience indicates that many obese primary care patients are interested in practice-based weight management programs. The challenge is to find the time and the resources to support these programs in the current and future fiscal environments.

\section{References}

1. Clinical guidelines on the identification, evaluation, and treatment of overweight and obesity in adults. NHLBI preprint. Bethesda, Md: National Institutes of Health, National Heart, Lung, and Blood Institute, June 1998.

2. White KL, Williams TF, Greenberg BG. The ecology of medical care. N Engl J Med 1961;265:885-92.

3. Wolf AM, Colditz GA. Current estimates of the economic cost of obesity in the United States. Obes Res 1998;6:97-106.

4. Frank A. Futility and avoidance: medical professionals in the treatment of obesity. JAMA 1993;269: 2132-3.

5. Kreuter MW, Scharff DP, Brennan LK, Lukwago $\mathrm{SN}$. Physician recommendations for diet and physical activity: which patients get advised to change? Prev Med 1997;26:825-33.

6. Logue E, Gilchrist V, Bourguet C, Bartos P. Recognition and management of obesity in a family prac- 
tice setting. J Am Board Family Pract 1993;5:45763.

7. Logue E, Smucker $W$, et al. Central obesity and beliefs regarding diet, exercise, weight loss in a primary care setting. 22nd Annual Meeting of the North American Primary Care Research Group, October 2-5, 1994, Toronto, Ontario, Canada.

8. Atkinson RL, Callaway CW, St Jeor S, Wolf-Novak L. A sane approach to weight loss. Patient Care 1995;Nov:152-80.

9. Polley BA, Jakicic JM, Venditti EM, Barr S, Wing $R R$. The effects of health beliefs on weight loss in individuals at high risk for NIDDM. Diabetes Care 1997;20:1533-8.

10. Fuller PR, Perri MG, Leermakers EA, Guyer LK. Effects of a personalized system of skill acquisition and an educational program in the treatment of obesity. Addict Behav 1998;23:97-100.

11. Perri MG, Sears SF Jr, Clark JE. Strategies for improving maintenance of weight loss. Toward a continuous care model of obesity management. Diabetes Care 1993;16:200-9.

12. Brownell $\mathrm{KD}$. Obesity: understanding and treating a serious, prevalent, and refractory disorder. J Consult Clin Psychol 1982;50:820-40.

13. Baum JG, Clark HB, Sandler J. Preventing relapse in obesity through posttreatment maintenance systems; comparing the relative efficacy of two levels of therapist support. J Behav Med 1991;14:287-302.

14. Prochaska JO. Strong and weak principles for progressing from precontemplation to action on the basis of twelve problem behaviors. Health Psychol 1994;13:47-51.

15. Alberti KG. A coordinated approach to treat chronic disorders. World Health Forum 1991;12:285-288.

16. Brownell KD, Wadden TA. The heterogeneity of obesity: fitting treatments to individuals. Behav Ther 1991;22:153-77.

17. Gilchrist V, Miller RS, Gillanders WR, et al. Does family practice at residency teaching sites reflect community practice? J Fam Pract 1993;37:555-63.

18. Objectives $1.2,2.3,15.10$, and 17.12 . Healthy people 2000: national health promotion and disease prevention objectives. Washington, DC: Public Health Service, 1990.

19. Bray GA. Pathophysiology of obesity. Am J Clin Nutr 1992;55(2 Suppl):488S-494S.

20. Prochaska JO, Norcross JC, DiClemente CC. Changing for good. New York: Avon Books, 1994.

21. Epi Info, version 6.03b. Stone Mountain, Ga: USD, Inc, 1994.

22. Teleform standard version 6.0. Vista, Calif: Cardiff Software, 1998.

23. Jacobs DR Jr, Elmer PJ, Gorder D, Hall Y, Moss D. Comparison of nutrient calculation systems. Am J Epidemiol 1985;121:580-92.

24. Sallis JF, Haskell WL, Wood PD, et al. Physical activity assessment methodology in the Five-City Project. Am J Epidemiol 1985;121:91-106.

25. Black AE, Prentice AM, Goldberg GR, et al. Measurements of total energy expenditure provide insights into the validity of dietary measurements of energy intake. J Am Diet Assoc 1993;9:572-9.

26. Jensen GL, Rogers J. Obesity in older persons. J Am Diet Assoc 1998;98:1308-11.

27. McDowell MA, Briefel RR, Alaimo K, et al. Energy and macronutrient intakes of persons ages 2 months and over in the United States: Third national health and nutrition examination survey, phase 1, 1988-91. Advance data from vital and health statistics; No 255. Hyattsville, Md: National Center for Health Statistics, 1994:1-24. 